\title{
Implementation of Telerehabilitation in Response to COVID-19: Lessons Learnt from Neurorehabilitation Clinical Practice and Education
}

Nada Signal PhD, MHSC(Hons), BHSC

Associate Professor of Physiotherapy and Associate Head of Research, School of Clinical Sciences, Auckland University of Technology, Auckland; New Zealand Senior Physiotherapist, Neuro Rehab Results Limited, Auckland, New Zealand

Tara Martin MHSC, BHSC(Hons)

Previous Clinical Lead, On the Go Physio, Christchurch, New Zealand

Adam Leys BHSC

Physiotherapist, Early Discharge and Rehabilitation Service, Waitematā District Health Board, Auckland, New Zealand

Rebecca Maloney BHSc, PGCert Leadership

Advanced Clinician Physiotherapist, Auckland District Health Board Community Services, Auckland, New Zealand

Felicity Bright PhD, MHSC, BSLT(Hons)

Senior Lecturer in Rehabilitation, School of Clinical Sciences, Auckland University of Technology, Auckland, New Zealand

\section{ABSTRACT}

The health response to the COVID-19 pandemic has had significant impacts on neurorehabilitation provision both internationally and in New Zealand. Telerehabilitation, the delivery of rehabilitation at a distance using information and communication technologies, was advocated as a means of addressing the rehabilitation needs of our patients while maintaining physical distancing and reducing the risk of viral transmission. Despite research evidence indicating that telerehabilitation is as effective as in-person rehabilitation for people with neurological conditions, there were significant challenges in delivering and sustaining telerehabilitation practice. We draw upon our experiences in delivering telerehabilitation in neurorehabilitation clinical practice and education to reflect on the process of practice change and to consider how these experiences can inform practice development in the future. We propose that rehabilitation organisations and physiotherapists continue to develop capability to provide telerehabilitation; that physiotherapists and the physiotherapy profession focus on translating their communicative, relational and clinical skills to the digital space to ensure they are competent in telerehabilitation; and that, as a profession, we focus on what constitutes "best practice" in telerehabilitation, and how in-person and telerehabilitation can be integrated to provide engaging, evidence-based and person-centred rehabilitation.

\section{Signal, N., Martin, T., Leys, A., Maloney, R. \& Bright, F. (2020). Implementation of telerehabilitation in response to COVID-19: Lessons learnt from neurorehabilitation clinical practice and education. New Zealand Journal of Physiotherapy, 48(3), 117-126. https://doi.org/10.15619/NZJP/48.3.03}

Key Words: Rehabilitation, Telerehabilitation, COVID-19, Physiotherapy, Education, Communication, Digital Health, Telehealth

\section{BACKGROUND}

Telerehabilitation is the delivery of rehabilitation across a distance using information and communication technologies. During telerehabilitation, communication between the healthcare professional and patient can be mediated by telephone, text messaging, email, web-based resources, videoconferencing, rehabilitation devices, and wearable technologies. Depending on the mode of delivery, communication can be synchronous (in real time) or asynchronous (where communication occurs with a delay, without the need to respond immediately) (Figure 1). A range of commercially available platforms have been developed to support the delivery of telerehabilitation, such as PhysiTrack $®$ and Cliniko®.

Telerehabilitation has a number of potential advantages over in-person rehabilitation. It can improve access to rehabilitation for those who live remote to healthcare services, and reduce monetary, time and environmental costs associated with travel to rehabilitation services (Smith et al., 2020; Soopramanien et al., 2020). Telerehabilitation can support the standardisation of delivery of care and information provision, promote selfmanagement and contextualisation of rehabilitation to the person's home and community environment, and help engage whānau ${ }^{1}$ in the rehabilitation process (Chen et al., 2019; Matamala-Gomez et al., 2020). It also offers the opportunity to effectively monitor rehabilitation outcomes using patient reported outcomes (Chen et al., 2019; Cramer et al., 2019; Knepley et al., 2020; Smith et al., 2020).

\footnotetext{
Whānau is the Māori term for extended family, family group, a familiar term of address to a number of people - the primary economic unit of traditional Māori society. In the modern context the term is sometimes used to include friends who may not have any kinship ties to other members (Moorfield, 2003-2020).
} 
Figure 1

Telerehabilitation Methods of Delivery

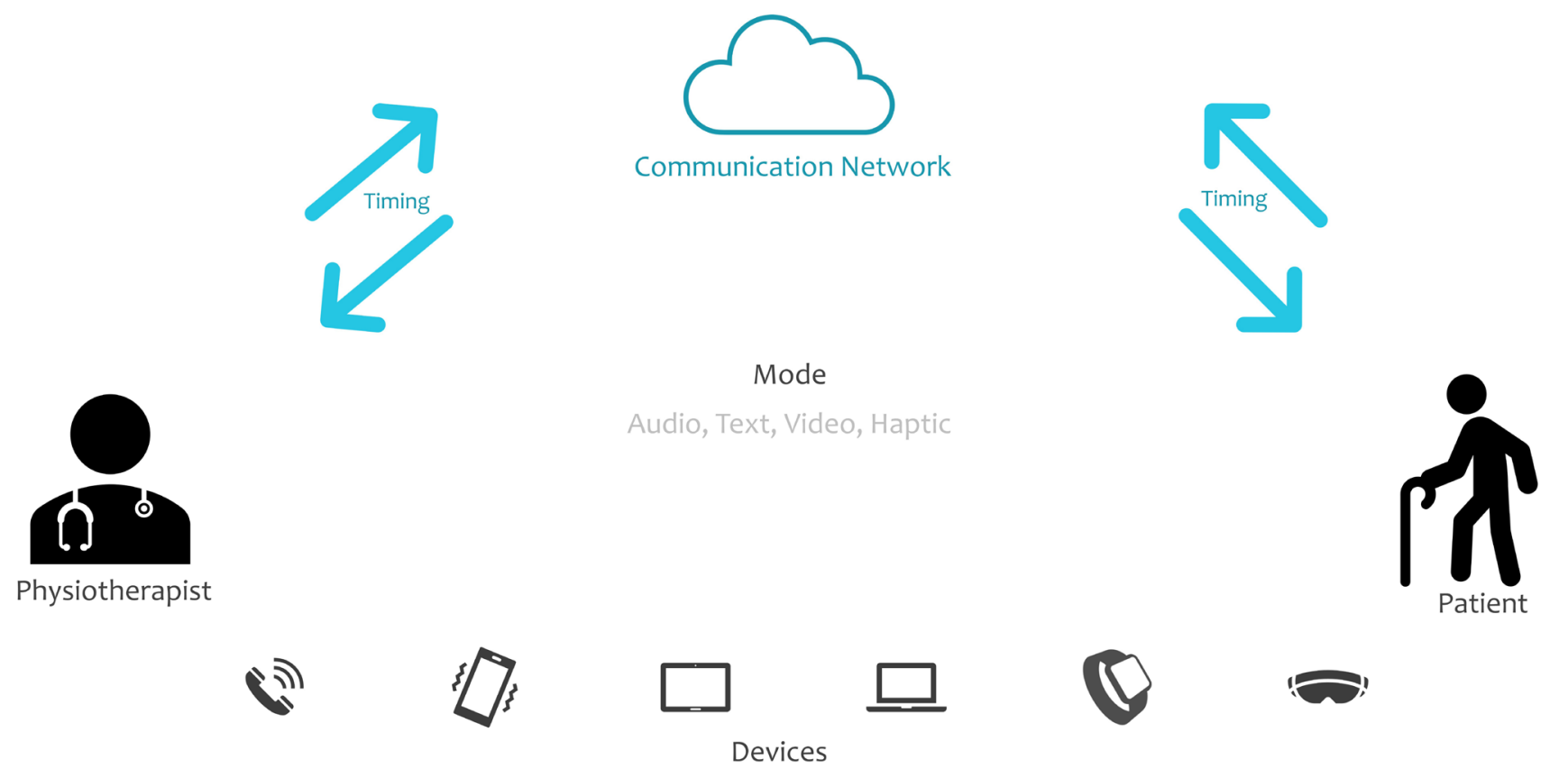

The evidence base supporting the effectiveness of telerehabilitation has grown over the last 15 years, with published randomised controlled trials for a range of neurological populations (for example, Cramer et al., 2019; Gandolfi et al., 2017; Rimmer et al., 2018; Saywell et al., 2020). For example, a 2020 Cochrane systematic review and metaanalysis found that telerehabilitation is as effective as in-person rehabilitation for people with stroke (Laver et al., 2020), with similar findings seen in other populations (Di Tella et al., 2020; Ownsworth et al., 2018). Telerehabilitation has been shown to have positive impacts on activities of daily living, healthrelated quality of life, and depressive symptoms in people with neurological disabilities (Appleby et al., 2019; Laver et al., 2020; Ownsworth et al., 2018). Preliminary evidence suggests that telerehabilitation may be more cost-effective than in-person interventions (Caughlin et al., 2020; Housley et al., 2016; Lloréns et al., 2015). It is important to note that systematic reviews and meta-analyses of telerehabilitation synthesise findings from interventions which use information and communication technologies to deliver rehabilitation remotely. Studies vary in the information and communication technologies used, and the mode and timing of communication. They also differ markedly in rehabilitation content, dosage, and theoretical underpinnings. Further work is required to identify what types of interventions are best delivered using telerehabilitation, when and how they are best delivered, for whom it is most appropriate, and how telerehabilitation approaches can effectively be integrated with in-person rehabilitation.

The health response to the COVID-19 pandemic has had significant impacts on rehabilitation provision (Aguiar de Sousa et al., 2020; Liu et al., 2020). In New Zealand, inpatient rehabilitation providers identified that the lockdowns during the COVID-19 pandemic led to (a) a shift of resources to focus on preparations for an anticipated surge in COVID-19 patients; (b) a reduction in the number of patients admitted to inpatient care; (c) suspension of rehabilitation in people with COVID-like symptoms; (d) a reduction in rehabilitation beds and staffing to accommodate physical distancing requirements; (e) limitations to the amount, type, and location of rehabilitation activities carried out; (f) restricted whānau involvement in rehabilitation; and (g) early, untimely and unsupported discharge of patients to home (New Zealand National Stroke Network, personal communication, April 16 and September 3, 2020). Yet, despite significant impacts on the provision of inpatient rehabilitation, the pandemic also resulted in the suspension of, or a marked reduction in, rehabilitation delivered through outpatient, community and residential care services (Bettger et al., 2020; Y. Ratnasabapathy \& J. Gundy, personal communication, September 2020). These limitations on service delivery reflected the need to prevent transmission of the virus amongst staff and vulnerable patients, and patient and healthcare providers' fear of transmission of the virus when engaging in rehabilitation. Internationally the impact of the pandemic has also seen rehabilitation services suspended as healthcare services have been overwhelmed by patients experiencing the acute effects of COVID-19 infection (Aguiar de Sousa et al., 2020; Leira et al., 2020; Willan et al., 2020). Prior to the pandemic, audits of rehabilitation provision in New Zealand consistently highlighted delays in service provision and limitations in the amount of rehabilitation provided (McNaughton et al., 2014; Thompson et al., 2019; Yeo et al., 2016). At this stage, it is not possible to estimate the compounding effect of the COVID-19 pandemic 
on access to neurorehabilitation, but given what we know about the importance of rehabilitation in reducing dependence, improving health-related quality of life and carer burden, we must mitigate this impact.

Telehealth has consistently been advocated as a means of providing healthcare services while maintaining physical distancing and reducing the risk of viral transmission during the COVID-19 pandemic. Yet, despite evidence of the effectiveness of telerehabilitation, its implementation in response to COVID-19 has been slow and challenging. In New Zealand, few district health boards (DHBs) achieved a substantial implementation of telerehabilitation in response to COVID-19 lockdowns, with provision often limited to telephone and email contact, and to a lesser extent, video conferenced rehabilitation sessions using tools such as Zoom $\circledast$ and Microsoft Teams $®$. The authors are not aware of any DHBs which adopted a commercially available telerehabilitation platform to support their service delivery. Private neurorehabilitation practices appeared to achieve a quicker transition to telerehabilitation, sometimes using commercially available telerehabilitation platforms. Many of these new methods of delivering rehabilitation were not sustained when public health strategies and physical distancing restrictions were relaxed, illustrating the challenges of delivering effective telerehabilitation, and the complexities of embedding and sustaining such a substantive change in healthcare practice. It is essential that, as a profession, we learn from this experience in order to prepare for future surges in COVID-19 and future infectious disease outbreaks, to enhance equitable access to rehabilitation, and to optimise the delivery of neurorehabilitation in general (Ford et al., 2020).
Aims

The purpose of this commentary is to reflect on the experience of rapidly implementing telerehabilitation in response to COVID-19 in neurorehabilitation clinical practice and physiotherapy education in New Zealand. In this commentary, we use our different experiences to reflect on the process of practice change and consider how these can inform practice development in the future. We draw upon our experience delivering telerehabilitation in private practice (TM, NS), in DHBs $(A L, R M)$, when educating student physiotherapists (FB, NS), and when supporting the professional development of practising physiotherapists upskilling in telerehabilitation ( $F B, A L, R M, T M$, NS). The commentary also draws upon published research and recently published editorials to support our reflections, inform our understanding, and to make suggestions for good practice moving forward.

\section{LESSONS LEARNT}

An overview of the key components of telerehabilitation is provided in Figure 2

\section{Organisational readiness}

Implementation of telerehabilitation occurred at a time of high stress and significant uncertainty, both in the workplace and in people's personal lives, which brought additional challenges to implementing a new way of working. In most cases, individuals and organisations began the implementation of telerehabilitation with little or no experience, and few resources. Swift implementation was supported by organisational readiness, in particular, where preparations for telerehabilitation were already underway, telehealth had been successfully used

\section{Figure 2}

Key Components of Telerehabilitation

\section{SETUP}

ASSESSMENT

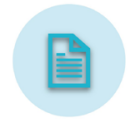

REFERRAL

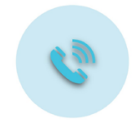

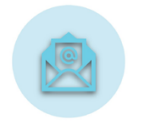

TECHNOLOGY SCREENING

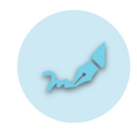

INFORMED CONSENT

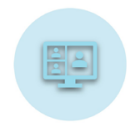

TECHNOLOGY TESTING

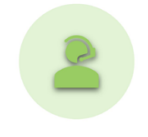

SUBJECTIVE ASSESSMENT

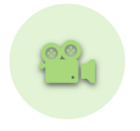

VIDEO OF FUNCTION

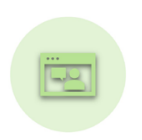

OBJECTIVE ASSESSMENT

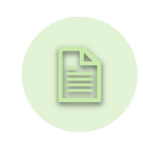

OUTCOME MEASUREMENT

\section{TREATMENT}

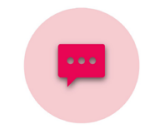

DEVELOPING A THERAPEUTIC RELATIONSHIP
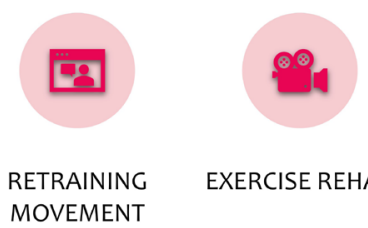

EXERCISE REHAB

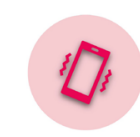

SELF MANAGEMENT

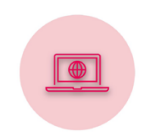

EDUCATION

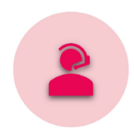

MANAGING SYMPTOMS 
in other healthcare services within the organisation, or staff had experience of telerehabilitation before the pandemic. A supportive and resourceful information technology (IT) department, strong support from organisational leaders and "on the ground" clinical champions enabled some organisations to adopt telerehabilitation quickly.

Technological tools used in providing telerehabilitation were in most instances selected based on platforms which organisations already had access to, as opposed to an evaluation of the needs of the patients and physiotherapists. Often physiotherapists did not have suitable workspaces or adequate hardware to support the efficient delivery of telerehabilitation. At a minimum, a web camera and speaker/microphone headset (ideally wireless), a second screen, and a strong, stable internet connection is required, along with access to email, a work telephone, and a private workspace.

Early in the implementation process, limited information and resources were available to support physiotherapists. At times, advice around COVID-19 alert level restrictions and their impact on healthcare delivery, and the medicolegal implications of delivering rehabilitation through information and communication technologies were contradictory and confusing. Not all physiotherapists were aware of their obligations under the Health Information Privacy Code and the Telecommunications Information Privacy Code, especially in relation to the selection of technology platforms. National and international networks and groups, such as Allied Health Aotearoa New Zealand, the New Zealand National Stroke Network, the NZ Telehealth Forum Resource Centre, and the Australian Telehealth for Stroke Community of Practice, quickly self-organised to crowdsource expertise, information and resources, as did commercially available telerehabilitation platforms. Many clinical champions engaged with and contributed to these networks, and a large and growing body of resources is now available (Allied Health Aotearoa New Zealand, 2018; Lee et al., 2020; Physiotherapy Board of New Zealand, 2020).

Effective delivery of telerehabilitation required physiotherapists to be competent managing the technical aspects of service delivery, not only for themselves but for their patients (Caughlin et al., 2020). A lack of technical competence was seen as a key barrier for many physiotherapists. This appeared to have a flow-on effect to communication and clinical skills. When physiotherapists were focused on how the technology worked or the activities they needed to complete, they appeared more likely to overlook the patient's experience, resulting in task-focused interactions. Having experience of different technologies, such as Zoom® or practice management software, helped some quickly upskill. Early adopters of telerehabilitation played an important role in advocating for telerehabilitation and supported colleagues to develop technical competence. Key elements which supported the development of technical competence included having documented, easy-to-follow procedures to guide the set-up, use and troubleshooting of technical issues experienced by physiotherapists and patients; active support from IT departments; creating a buddy system between early adopters and less technically competent staff; addressing technical issues and solutions in team meetings; and devoting time to mastering the platform through dedicated practice before working with patients.

\section{TIPS}

- Select technology tools and platforms which meet the needs of all users.

- Ensure staff have suitable hardware and space.

- Link with telerehabilitation networks to share expertise, information and resources.

- Identify clinical champions.

- Develop 'How to..." guides.

- Practice, practice, practice..

- Focus team and professional development activities on telerehabilitation.

\section{Getting the patient set up}

Telehealth is a new model of healthcare delivery, and consequently, most patients were unfamiliar with it. This meant that physiotherapists needed to "pitch" the concept of telerehabilitation, clearly describing what was involved and the potential benefits and limitations. At times, patients and whānau were sceptical of the value of telerehabilitation, but for many, once they had experienced it, they were able to see its value in rehabilitation.

Technology screening was required to determine the patient's access to a suitable device and data connection; their experience using technology and social media; their capacity to engage in telephone, email, text message and videoconferenced interactions; and the level of whānau support and resources. In some cases, physiotherapists needed to outline the privacy and data security concerns associated with platforms such as FaceTime ${ }^{\circledR}$ and WhatsApp $\AA$ to explain why they were not suitable for telerehabilitation. One DHB offered the loan of iPads with data SIM cards, enabling those without digital access to engage in telehealth services. When the telerehabilitation pitch and technology screening was undertaken by a third party or a staff member who was sceptical about the appropriateness, efficacy or value of telerehabilitation, it was often unsucessful. At times, it was noted that healthcare professionals were reluctant to offer telerehabilitation to people who were older, communicatively or cognitively impaired, from diverse cultural backgrounds, or those experiencing financial hardship. Healthcare professionals sometimes made assumptions about the person's capacity, preferences and resources for telerehabilitation. It was not uncommon for these assumptions to be incorrect. Many patients and their whānau were able to access a suitable device and connection, and effectively engage in telerehabilitation. Some organisations developed innovative ways of pitching and preparing people for telerehabilitation, including videoconferencing with patients before discharge from inpatient care with the support of the inpatient physiotherapist, having a physiotherapy assistant familiar with telerehabilitation 
provide the pitch, and getting whānau and friends onboard before pitching to the patient. Some private providers also offered a free first session for patients to trial telerehabilitation before they committed to using it.

The telerehabilitation set-up worked most effectively when the physiotherapist devoted the first session to supporting the patient and whānau to develop competence with the technology platform and to optimise their operating system, data connectivity, audio and video quality, and environmental set-up for future sessions. Establishing and documenting camera and patient position for different rehabilitation tasks during this session saved set-up time in future sessions.

\section{TIPS}

- Assume that everyone can engage in some aspects of telerehabilitation with support.

- Pitch telerehabilitation to all patients.

- Devote time to setting the patient up for telerehabilitation.

- Test out camera and patient positions during the set up phase.

Translating communication and relational skills to the digital space

Communication is an area of practice that is commonly taken for granted and often appears to work well with little conscious attention. The shift to telerehabilitation challenged this and highlighted the many complexities associated with communicating via technology. The changes in nonverbal communication, loss of touch and disruptions to usual conversational flow due to connectivity lags all impacted on how patients and physiotherapists related and worked together. Turn-taking was less natural, with more likelihood of overlapping speech. Facial expressions, which might ordinarily communicate emotions or the desire to speak, were less obvious in a video consultation or not evident at all in a telephone consultation. To mitigate this, physiotherapists made turntaking opportunities overt, clarified the patient's understanding frequently, and used purposeful eye contact and exaggerated facial expression and hand gestures. Our experiences echoed the call to "step up" verbal and non-verbal skills (Graham, 2020), adapt communication to build strong therapeutic relationships, ensure patient understanding, facilitate engagement, and monitor the subtle aspects of communication and well-being that might easily be missed.

Physiotherapists expressed concerns that telerehabilitation might negatively impact the therapeutic relationship, although research suggests the same concerns are not generally held by patients (Lawford et al., 2019). Once using telerehabilitation, physiotherapists indicated that building and maintaining relationships went more smoothly than expected and the overall strength of the relationship was, from their perspective, unchanged. However, they noted that it could take longer to build therapeutic relationships and required an intentional focus. This process was naturally more straightforward when the physiotherapist knew the patient. When there was no preexisting relationship, physiotherapists needed to spend more time getting to know not just the patient, but the context in which rehabilitation was occurring. Allowing for "relational transitions" between checking the technology set-up and rehabilitation interventions was important. Moving forward, the Hui Process may provide a helpful framework for all (Lacey et al., 2011). This presents four stages of a clinical interaction: mihimihi (the initial greeting and engagement, which could include the checking of technology), whakawhānaungatanga (building relationships and making connections), kaupapa (attending to the clinical purpose of the interaction), and poroaki (closing the session). We noted that telerehabilitation changed the power dynamic of the therapeutic relationship, creating a more balanced relationship between the patient and physiotherapist; this has been identified in previous telerehabilitation research (Bridges Self Management, 2020; Lawford et al., 2019). A range of factors appeared to give patients greater control over the rehabilitation process, such as patients being in their own environment, physiotherapists being less able to "impose" expertise through touch, and patients having the ability to choose to disengage if their needs and priorities were not being met (Graham, 2020; Lawford et al., 2019).

\section{TIPS}

- Allow time for relational, pro-social talk before and within rehabilitation activities.

- Notice and acknowledge emotions evident in verbal and non-verbal communication.

- Emphasise empathetic behaviours such as nodding, gestures and facial expression.

- Voice empathy and acknowledge the patient's perspective and experience.

Working with patients with cognitive and communication impairments, and those from non-English speaking backgrounds presented unique challenges. Communication strategies that we might intuitively use during in-person rehabilitation, such as touch, facilitation of movement, and demonstrations, are either not available or are more challenging. Interdisciplinary teamwork was vital, aided when speech-language therapists saw patients first and developed supported communication resources such as communication books. That said, simple devices like pen and paper, and whiteboards were invaluable, enabling patients and therapists to write key words and draw diagrams, aiding comprehension and expression. Whānau members assisted communication, although care needed to be taken to ensure the physiotherapist did not exclude the patient by only talking with whānau. The inclusion of a third party, such as an interpreter, presented particular challenges, as the physiotherapist sometimes needed to coach other care providers in the use of technology and monitor their adherence to data security and privacy requirements. 
TIPS

- Connect with the speech-language therapist.

- Involve whānau.

- Use a physical or electronic whiteboard to support communication.

Translating clinical skills to the digital space Whilst the research evidence base indicates that telerehabilitation is as effective as in-person rehabilitation, physiotherapists expressed concerns about the implications of working in the digital space. Many of these concerns centred on the inability to touch, physically support and facilitate movement. However, telerehabilitation highlighted the need to employ a range of skills during rehabilitation which do not rely on touch or physical contact. The rehabilitative emphasis shifted to task-specific training, exercise rehabilitation, and activity promotion. It often involved a coaching approach, with greater emphasis on problem-solving, patient empowerment, and education; these approaches all helped build capability for selfmanagement (Hinman et al., 2019; Lawford et al., 2019). This shift from "doing to" to "working with" the person required therapists to seek out, rely on and respond to the patient's expertise. This shift in ways of working also sometimes required physiotherapists to reflect on their professional identity and scope of practice.

\section{Assessment}

Telerehabilitation required a change in the timing and mode of information collection during the assessment process. Assessment was often distributed over three to six shorter interactions and was completed using telephone, email, online forms, prerecorded videos of function, and videoconferencing. Many physiotherapists valued this, noting that telerehabilitation (a) supported clinical reasoning, particularly for novice practitioners for whom the pressure of a single assessment session is sometimes challenging; and (b) mitigated fatigue in patients who could not tolerate a single long assessment session. Some physiotherapists described how conducting the assessment in the digital space enabled them to work more efficiently. Automating the delivery of instructions, having the patient email videos of functional tasks and objective assessments, and collecting patient-reported outcome measures all reduced face-to-face time. Well-structured, holistic documentation templates, often completed by physiotherapists in real time during telerehabilitation, helped ensure that all areas important in the assessment were covered, therefore reducing documentation time. This sometimes made it easier to raise sensitive subjects, such as sexuality. When embedded in the template, sensitive subjects were addressed as simply another area that needed to be discussed. When not using a telerehabilitation platform or documentation template, it was important for physiotherapists to carefully plan the assessment process to maximise the quality and quantity of information gleaned from each interaction with the patient.

Assessment in telerehabilitation relied heavily on subjective interviewing, patient-reported outcome measurement, and functional assessment as the primary sources of information to support clinical reasoning. It was often not possible to conduct objective assessment at the impairment level, for example, of muscle tone or sensation, due to the hands-on nature of these assessment techniques. Instead, therapists needed to interpret subjective interview findings and movement observation to identify relevant impairments. This posed particular challenges to novice and student physiotherapists, whose clinical reasoning is not always supported by a strong understanding of the relationship between symptoms, participation, functional activity limitations, impairment, and pathophysiology. Observation of movement was influenced by video quality, meaning that optimising video and audio quality was essential. Coaching patients and whānau in videography became a core skill to support both assessment and treatment.

One advantage of telerehabilitation was the ease with which patient-reported outcomes could be gathered. However, some physiotherapists and student physiotherapists expressed concern that these types of measures are less valid than objective outcome measurements. This may reflect underlying beliefs that our movement observation and physical assessment skills are more reliable than the patient's account of their experiences or functioning. However, this is not supported by evidence (Hinman et al., 2019). Additionally, therapists should be reassured by evidence from musculoskeletal practice which suggests that diagnostic accuracy in telerehabilitation assessment is equivalent to in-person assessment (Richardson et al., 2017; Russell et al., 2010). We suggest that this different approach to information gathering helps redistribute power in the therapeutic relationship and better recognises the expertise that patients hold and bring to rehabilitation.

\section{TIPS}

- Plan the timing and mode of data collection across the assessment process.

- Gather information using online forms and email communication.

- Ask the patient to email videos of key functional activities.

- Use a template to support documentation in real time.

- Source and use patient reported outcome measures.

\section{Treatment}

One marked advantage of telerehabilitation is that it occurs in the person's own home or community. Contextualised and meaningful practice is often difficult to create in clinical environments, yet it is known to increase the likelihood that learning is retained and that skills transfer to everyday life (Kleim \& Jones, 2008). However, without the accoutrements of clinical spaces, physiotherapists had to be resourceful, for example, using washing baskets and backpacks filled with books as strength training equipment and kitchen benches as parallel bars. Telerehabilitation also supported whānau engagement in rehabilitation, either through direct involvement in videoconferencing or inclusion in telephone, text and email communication. 
A key concern for physiotherapists was safety, particularly when patients were undertaking balance and walking rehabilitation activities. Similar to the approaches taken during in-person community rehabilitation, physiotherapists mitigated safety risks through careful planning, communication, and monitoring.

\section{TIPS}

- Establish and document emergency procedures for both the physiotherapist and patient.

- Provide written and pictorial instructions detailing safe exercising environments, clothing, footwear and equipment requirements.

- Have the patient to scan the camera around the room to check for hazards and ensure privacy.

- Have the patient work near solid walls and furniture such as kitchen benches, in corners, hallways, adjacent to beds and using mobility aids as appropriate.

- Ensure that the patient can get up off the floor if they do lose their balance.

Safety also relied on the physiotherapist's ability to develop a rehabilitation session which progressively developed mastery of movement skills. Having a repertoire of progressions and modifications tailored to the patient within the session plan and explicitly seeking regular feedback from the patient about the difficulty level of each task was essential in effectively titrating difficulty. This required precise communication and was most successful when a structured approach was taken. Cues were often needed to paint a clear picture for the patient, drawing on analogies (e.g. "Stand tall like a tree"), everyday contexts (e.g. "Show me how you would shampoo the back of your head"), and environmental cues (e.g. "Step toward the TV"). Physically disabled patients who required physical support to achieve sitting or standing presented particular challenges. Enlisting whānau and caregivers as assistants required careful planning and education, and constant monitoring of what the whānau member was feeling and how much they were assisting.

\section{TIPS}

- Send a description of the session structure in advance.

- Take a structured approach to movement coaching o Physically demonstrate the movement.

o Specify and confirm the starting position.

o Add movement and task variations one at a time.

o Reinforce each step through gesture and verbal affirmations.

- Follow up with key 'take home' messages in an email or text after the session.

Physiotherapists described using multiple modes of communication, and tailoring both the mode and timing of the communication to the patient and their whānau. In addition to videoconferenced sessions, physiotherapists saw value in emailing instructions and session plans in advance, using text reminders to support attendance and engagement in exercise programmes, emailing or providing links to educational content, and developing and emailing videos of exercises and rehabilitation tasks. These strategies supported patients in home exercise programmes and self-management, and allowed physiotherapists to tailor education to the person. However, for some, substantial time went into sourcing, developing, and individualising resources, and few available resources were considered culturally or communicatively accessible. This process was streamlined where organisations collaborated to develop and share resources, or physiotherapists used commercially available telerehabilitation platforms with already developed content and the ability to upload their own content.

Professional development

Telerehabilitation offered a powerful opportunity to embed student supervision, critical self-reflection and professional supervision into clinical practice for student physiotherapists, physiotherapists and physiotherapy assistants alike. Connecting colleagues into sessions through videoconferencing enabled specialists or other members of the interdisciplinary team to offer brief consultations related to specific issues. With the patient's consent, it was relatively easy to screen capture the session for later review or to include a third party in a video conference session to enable professional supervision. This also allowed physiotherapists to reflect on their intervention and their communication. However, it was noted that like other professional development opportunities, therapists were sometimes reluctant to engage in this shared practice and professional supervision with colleagues, and thus did not make the most of the opportunities telerehabilitation offers.

\section{DISCUSSION}

Telerehabilitation is a viable and effective approach to neurorehabilitation. It allows physiotherapists to provide larger doses of rehabilitation, which are essential to maximising recovery (Lohse et al., 2014). During the COVID-19 pandemic, telerehabilitation was valued when in-person rehabilitation was not an option. Indeed, some aspects of telerehabilitation were highly valued and offered advantages over in-person rehabilitation, highlighting that telerehabilitation should remain within our therapeutic repetoire. While telerehabilitation was being considered within many services and was in use in a small number of neurorehabilitation services in New Zealand prior to the pandemic, COVID-19 restrictions facilitated therapists and services to make a rapid shift in practice. Therapists and services invested significant time in upskilling, creating systems and structures, and developing resources to support the delivery of telerehabilitation. It is now critical to build on the experiences and investment to date, and the skills that therapists have developed, and to embed telerehabililitation in everyday neurorehabilitation so that patients, therapists, and services alike can fully benefit from the opportunities that telerehabilitation opens up. Moving into the future, we have opportunities to integrate in-person rehabilitation and telerehabilitation, using different modalities at different times, to maximise our ability to provide evidence-based, person-centred neurorehabilitation. 
The required and rapid shift to telerehabilitation challenged a number of our assumptions about both telerehabilitation and physiotherapy. Telerehabilitation has commonly been seen as the poor cousin to in-person rehabilitation. However, this experience has highlighted some of what telerehabilitation can offer to patients and physiotherapists - increasing dose, reducing travel time and cost, facilitating regular points of connection through the use of different modes of communication, and shifting of power. Our assumptions about who might struggle to engage with telerehabilitation, such as older people or Māori whānau (for whom kanohi ki te kanohi is important in relationship building) were not necessarily correct. That said, there is still significant work to be done to understand what engaging, highquality rehabilitation involves for different patient groups and whether there are inequities in access to, or quality of, service. We do not claim to fully understand how different patient groups have experienced telerehabilitation, and we suspect there will be significant differences across patient groups, particularly for marginalised and under-resourced populations. Developing better knowledge of the needs of these people and shaping services to best support their needs is imperative if we are to ensure equity in access, experience, and outcomes in rehabilitation on a long-term basis.

Implementing telerehabilitation and our response to this new way of working has also highlighted some of the deeper assumptions that underpin physiotherapy and rehabilitation practice. Touch and hands-on treatment are important components of physiotherapy identity, and the loss of this communication and clinical medium presented significant challenges. In line with published research on telerehabilitation, physiotherapists perceived the loss of touch and reliance on verbal communication to be potentially problematic and less effective (Lawford et al., 2019). This view reflects the historical underpinnings of physiotherapy practice, something that continues to be reinforced in education and practice (Moffatt and Kerry, 2018). Physiotherapists had to enhance their communication skills rapidly, and appreciate and attend to the therapeutic relationship. These are aspects of practice that are often taken for granted (Hinman et al., 2019), yet have been shown to be important factors in patient experience and outcomes (Pinto et al., 2012). Reliance on patient self-report has also challenged our understandings of whose knowledge is privileged. The unintended impact of telerehabilitation was a focus on self-management, and enhancing people's capability and confidence to self-manage (Bridges Self Management, 2020; Hinman et al., 2019; Lawford et al., 2019), areas emphasised within practice guidelines yet not consistently addressed well in clinical practice (Mudge et al., 2014). Together, these factors show how telerehabilitation can open up different ways of thinking about and doing neurorehabilitation. This is likely to be to the advantage of our patients and their whānau.

We need to integrate telerehabilitation into "usual practice", and COVID-19 has helped in this process. However, moving forward, there are a number of areas for educators, physiotherapists and services to consider. We have an obligation to develop skills and knowledge in telerehabilitation to ensure we meet the needs of our patients. Telerehabilitation should be a core competency of physiotherapy practice, part of undergraduate education, and a focus of professional development. To date, we have done the best possible in the circumstances, but we now need to consider what constitutes best practice telerehabilitation in New Zealand, and alongside this, what constitutes best practice neurorehabilitation with a blending of in-person and telerehabilitation. Our position is that best practice neurorehabilitation should include both in-person and telerehabilitation, not one or the other. This requires strong clinical reasoning to determine the aspects of each approach that are most appropriate and effective for which patients, and at what points and for which purposes within the rehabilitation process. There are opportunities to share and standardise resources and care pathways throughout the country, facilitating more efficient and effective ways of working. We also argue that services and structures, including reporting and financial systems, need to recognise the changing service delivery models of neurorehabilitation to ensure integrated neurorehabilitation services are appropriately funded and able to be provided. Quality frameworks and associated quality indicators, often unseen influences on practice, must be flexible to allow for multiple modalities of rehabilitation provision, rather than privileging in-person rehabilitation, regardless of whether or not it is the most appropriate for the patient and their needs.

\section{CONCLUSION}

Along with its many challenges, the COVID-19 pandemic has offered the physiotherapy profession an exceptional learning experience. Through the process of rapidly implementing telerehabilitation in neurorehabilitation clinical practice and education we have had the opportunity to learn new skills, relate to our patients in new ways, and reflect upon our professional identity and the future of rehabilitation practice in New Zealand. Whilst there are many opportunities to further develop telerehabilitation practice in New Zealand, our experiences in teaching and providing telerehabilitation over the last six months highlights that the most important thing is to start - give telerehabilitation a try, seek feedback from patients and their whānau, critically reflect on your practice, and don't be afraid to add telerehabilitation to your rehabilitation repertoire.

\section{DISCLOSURES}

No funding was received for this research. There are no conflicts of interest which may be perceived to interfere with or bias this study.

\section{PERMISSIONS}

Nada Signal gives permission for the use of the attached figures.

\section{ACKNOWLEDGEMENTS}

We acknowledge the patients and students we have worked with as we started implementing telerehabilitation in our clinical practice and education. Patients and whānau patiently worked with us as we all developed our competency in telerehabilitation. We also acknowledge colleagues, both in New Zealand and internationally, who have willingly shared their experiences, resources, and expertise. These often informal networks have been critical in enhancing practice and teaching, and helping ensure people continued to receive high-quality rehabilitation during the COVID-19 pandemic. 


\section{ADDRESS FOR CORRESPONDENCE}

Nada Signal, School of Clinical Sciences, Auckland University of Technology, 90 Akoranga Drive, Northcote, Auckland, New Zealand.

Email: nada.signal@aut.ac.nz

\section{REFERENCES}

Aguiar de Sousa, D., van der Worp, H. B., Caso, V., Cordonnier, C., Strbian, D., Ntaios, G., Schellinger, P. D., Sandset, E. C.; European Stroke Organisation. (2020). Maintaining stroke care in Europe during the COVID-19 pandemic: Results from an international survey of stroke professionals and practice recommendations from the European Stroke Organisation. European Stroke Journal, 5(3), 230-236. https://doi. org/10.1177/2396987320933746

Allied Health Aotearoa New Zealand. (2018). Allied health best practice guide for telehealth. https://www.alliedhealth.org.nz/uploads/8/8/9/4/88944696/ best_practice_guide_for_telehealth.pdf

Appleby, E., Gill, S. T., Hayes, L. K., Walker, T. L., Walsh, M., \& Kumar, S (2019). Effectiveness of telerehabilitation in the management of adults with stroke: A systematic review. Plos One, 14(11), e0225150. https://doi. org/10.1371/journal.pone.0225150

Bettger, J. P., Thoumi, A., Marquevich, V., De Groote, W., Battistella, L. R. Imamura, M., Ramos, V. D., Wang, N., Dreinhoefer, K. E., Mangar, A., Ghandi, D. B. C., Ng, Y. S., Lee, K. H., Tan, J. T. M., Pua, Y. H., Inzitari, M., Mmbaga, B. T., Shayo, M. J., Brown, D. A. . . Stein, J. (2020). COVID-19: Maintaining essential rehabilitation services across the care continuum. BMJ Global Health, 5, e002670. https://doi.org/10.1136/ bmjgh-2020-002670

Bridges Self Management. (2020). Community team guidance: Remote conversations that support self-management. https://drive.google.com/ file/d/1r4JBbBHaZcOmPCezerW1_YjTi1g7zAU1/view

Caughlin, S., Mehta, S., Corriveau, H., Eng, J. J., Eskes, G., Kairy, D., Meltzer, J., Sakakibara, B. M., \& Teasell, R. (2020). Implementing telerehabilitation after stroke: Lessons learned from Canadian trials. Telemedicine and e-Health, 26(6), 710-719. https://doi.org/10.1089/tmj.2019.0097

Chen, Y., Abel, K. T., Janecek, J. T., Chen, Y., Zheng, K., \& Cramer, S. C. (2019). Home-based technologies for stroke rehabilitation: A systematic review. International Journal of Medical Informatics, 123, 11-22. https:// doi.org/10.1016/j.jimedinf.2018.12.001

Cramer, S. C., Dodakian, L., Le, V., See, J., Augsburger, R., McKenzie, A., Zhou, R. J., Chiu, N. L., Heckhausen, J., Cassidy, J. M., Scacchi, W., Smith, M. T., Barrett, A. M., Knutson, J., Edwards, D., Putrino, D., Agrawal, K., Ngo, K., Roth, E. J., . . . Janis, S.; National Institutes of Health StrokeNet Telerehab Investigators. (2019). Efficacy of home-based telerehabilitation vs in-clinic therapy for adults after stroke: A randomized clinical trial. JAMA Neurology, 76(9), 1079-1087. https://doi.org/10.1001/ jamaneurol.2019.1604

Di Tella, S., Pagliari, C., Blasi, V., Mendozzi, L., Rovaris, M., \& Baglio, F. (2020) Integrated telerehabilitation approach in multiple sclerosis: A systematic review and meta-analysis. Journal of Telemedicine and Telecare, 26(7-8), 385-399. https://doi.org/10.1177/1357633X19850381

Ford, G. A., Hargroves, D., Lowe, D., Rooney, G., Fisher, R., Oatley, H., \& Lough, J. (2020). Restoration and recovery of stroke services during the COVID-19 pandemic. https://www.oxfordahsn.org/wp-content/ uploads/2020/07/Restoration-and-recovery-of-stroke-services-during-theCOVID-19-pandemic-July-2020-1.pdf

Gandolfi, M., Geroin, C., Dimitrova, E., Boldrini, P., Waldner, A., Bonadiman, S., Picelli, A., Regazzo, S., Stirbu, E., \& Primon, D., Bosello, C., Gravina, A. R., Peron, L., Trevisan, M., Carreño Garcia, A., Menel, A., Bloccari, L., Valè, N., Saltuari, L., . . Smania, N. (2017). Virtual reality telerehabilitation for postural instability in Parkinson's disease: A multicenter, single-blind, randomized, controlled trial. BioMed Research International, 2017 7962826. https://doi.org/10.1155/2017/7962826
Graham, F. (2020, March 24). Telehealth rehabilitation overview session 1. YouTube. https://www.youtube.com/watch?v=mCqJclmha9g\&ct=t\%28EM AIL_CAMPAIGN_2_5_2020_16_2_COPY_01\%29

Hinman, R. S., Lawford, B. J., \& Bennell, K. L. (2019). Harnessing technology to deliver care by physical therapists for people with persistent joint pain: Telephone and video-conferencing service models. Journal of Applied Biobehavioral Research, 24(2), e12150. https://doi.org/10.1111/jabr.12150

Housley, S. N., Garlow, A. R., Ducote, K., Howard, A., Thomas, T., Wu, D. Richards, K., \& Butler, A. J. (2016). Increasing access to cost effective home-based rehabilitation for rural veteran stroke survivors. Austin Journal of Cerebrovascular Disease \& Stroke, 3(2), 1-11. https://www.ncbi.nlm. nih.gov/pmc/articles/PMC5175468/

Kleim, J. A., \& Jones, T. A. (2008). Principles of experience-dependent neural plasticity: Implications for rehabilitation after brain damage. Journal of Speech, Language, and Hearing Research, 51(1), S225-S239. https://doi. org/10.1044/1092-4388(2008/018)

Knepley, K. D., Mao, J. Z., Wieczorek, P., Okoye, F. O., Jain, A. P., \& Harel, N. Y. (2020). Impact of telerehabilitation for stroke-related deficits. Telemedicine and e-Health. Advance online publication. https://doi. org/10.1089/tmj.2020.0019.

Lacey, C., Huria, T., Beckert, L., Gilles, M., \& Pitama, S. (2011). The Hui Process: A framework to enhance the doctor-patient relationship with Māori. New Zealand Medical Journal, 124(1347), 72-78.

Laver, K. E., Adey-Wakeling, Z., Crotty, M., Lannin, N. A., George, S., \& Sherrington, C. (2020). Telerehabilitation services for stroke. The Cochrane Database of Systematic Reviews, 1, CD010255. https://doi. org/10.1002/14651858.CD010255.pub3

Lawford, B. J., Delany, C., Bennell, K. L., \& Hinman, R. S. (2019). "I was really pleasantly surprised": Firsthand experience and shifts in physical therapist perceptions of telephone-delivered exercise therapy for knee osteoarthritis-A qualitative study. Arthritis Care \& Research, 71(4), 545-557. https://doi.org/10.1002/acr.23618

Lee, A., Finnin, K., Holdsworth, L., Millette, D., \& Peterson, C.; Digital Physical Therapy Task Force. (2020). Report of the World Confederation for Physical Therapy/International Network of Physiotherapy Regulatory Authorities Digital Physical Therapy Practice Task Force. https://world.physio/sites/ default/files/2020-06/WCPT-INPTRA-Digital-Physical-Therapy-Practice-Taskforce-March2020.pdf

Leira, E. C., Russman, A. N., Biller, J., Brown, D. L., Bushnell, C. D., Caso, V., Chamorro, A., Creutzfeldt, C. J., Cruz-Flores, S., \& Elkind, M. S., Fayad, P., Froehler, M. T., Goldstein, L. B., Gonzales, N. R., Kaskie, B., Khatri, P., Livesay, S., Liebeskind, D. S., Majersik, J. J., . . . Worrall BB. (2020). Preserving stroke care during the COVID-19 pandemic: Potential issues and solutions. Neurology, 95(3), 124-133. https://doi.org/10.1212/ WNL.0000000000009713

Liu, R., Zhao, J., \& Fisher, M. (2020). The global impact of COVID-19 on acute stroke care. CNS Neuroscience \& Therapeutics, 26(10), 1103-1105 https://doi.org/10.1111/cns.13442

Lloréns, R., Noé, E., Colomer, C., \& Alcañiz, M. (2015). Effectiveness, usability, and cost-benefit of a virtual reality-based telerehabilitation program for balance recovery after stroke: A randomized controlled trial. Archives of Physical Medicine and Rehabilitation, 96(3), 418-425.e2. https://doi.org/10.1016/j.apmr.2014.10.019

Lohse, K. R., Lang, C. E., \& Boyd, L. A. (2014). Is more better? Using metadata to explore dose-response relationships in stroke rehabilitation. Stroke, 45(7), 2053-2058. https://doi.org/10.1161/ STROKEAHA. 114.004695

Moorfield, J. C.(2003-2020). Te Aka online Māori dictionary. https:// maoridictionary.co.nz/search?idiom $=$ \&phrase $=\&$ proverb=\&loan $=\&$ histLoan Words=\&keywords=whanau

Matamala-Gomez, M., Maisto, M., Montana, J. I., Mavrodiev, P. A., Baglio, F., Rossetto, F., Mantovani, F., Riva, G., \& Realdon, O. (2020). The role of engagement in teleneurorehabilitation: A systematic review. Frontiers in Neurology, 11, 354. https://doi.org/10.3389/fneur.2020.00354 
McNaughton, H., McRae, A., Green, G., Abernethy, G., \& Gommans, J. (2014). Stroke rehabilitation services in New Zealand: A survey of service configuration, capacity and guideline adherence. New Zealand Medical Journal, 127(1402), 10-9.

Moffatt, F., \& Kerry, R. (2018). The desire for "hands-on" therapy-a critical analysis of the phenomenon of touch. In B. E. Gibson, D. A. Nicholls, J. Setchell, \& K. S. Groven (Eds.), Manipulating practices: A critical physiotherapy reader (pp. 174-193). Cappelen Damm Akademisk. https:// doi.org/10.23865/noasp.29

Mudge, S., Stretton, C., \& Kayes, N. (2014). Are physiotherapists comfortable with person-centred practice? An autoethnographic insight. Disability and Rehabilitation, 36(6), 457-463. https://doi.org/10.3109/09638288.2013. 797515

Ownsworth, T., Arnautovska, U., Beadle, E., Shum, D. H., \& Moyle, W. (2018). Efficacy of telerehabilitation for adults with traumatic brain injury: A systematic review. Journal of Head Trauma Rehabilitation, 33(4), E33E46. https://doi.org/10.1097/HTR.0000000000000350

Physiotherapy Board of New Zealand. (2020). Telehealth standard. https:// www.physioboard.org.nz/standards/physiotherapy-standards/telehealthstandard

Pinto, R. Z., Ferreira, M. L., Oliveira, V. C., Franco, M. R., Adams, R., Maher, C. G., \& Ferreira, P. H. (2012). Patient-centred communication is associated with positive therapeutic alliance: A systematic review. Journal of Physiotherapy, 58(2), 77-87. https://doi.org/10.1016/S18369553(12)70087-5

Richardson, B. R., Truter, P., Blumke, R., \& Russell, T. G. (2017). Physiotherapy assessment and diagnosis of musculoskeletal disorders of the knee via telerehabilitation. Journal of Telemedicine and Telecare, 23(1), 88-95. https://doi.org/10.1177/1357633X15627237

Rimmer, J. H., Thirumalai, M., Young, H.-J., Pekmezi, D., Tracy, T., Riser, E., \& Mehta, T. (2018). Rationale and design of the tele-exercise and multiple sclerosis (TEAMS) study: A comparative effectiveness trial between a clinicand home-based telerehabilitation intervention for adults with multiple sclerosis (MS) living in the deep south. Contemporary Clinical Trials, 71 186-193. https://doi.org/10.1016/j.cct.2018.05.016
Russell, T., Truter, P., Blumke, R., \& Richardson, B. (2010). The diagnostic accuracy of telerehabilitation for nonarticular lower-limb musculoskeletal disorders. Telemedicine and e-Health, 16(5), 585-594. https://doi. org/10.1089/tmj.2009.0163

Smith, E. E., Mountain, A., Hill, M. D., Wein, T. H., Blacquiere, D., Casaubon, L. K., Linkewich, E., Foley, N., Gubitz, G., Simard, A. \& Lindsay, M. P. (2020). Canadian stroke best practice guidance during the COVID-19 pandemic. Canadian Journal of Neurological Sciences, 47(4), 474-478. https://doi.org/10.1017/cjn.2020.74

Saywell, N. L., Vandal, A. C., Mudge, S., Hale, L., Brown, P., Feigin, V. Hanger, C., \& Taylor, D. (2020). Telerehabilitation after stroke using readily available technology: A randomized controlled trial. Neurorehabilitation and Neural Repair. Advance online publication. https://doi. org/10.1177/1545968320971765

Soopramanien, A., Jamwal, S., \& Thomas, P. W. (2020). Digital health rehabilitation can improve access to care in spinal cord injury in the UK: A proposed solution. International Journal of Telerehabilitation, 12(1), 3-15. https://doi.org/10.5195/ijt.2020.6312

Thompson, S., Douwes, J., Barber, A., Cadilhac, D., McNaughton, H., Gommans, J., Fink, J., Davis, A., Feigin, V., \& Ranta, A. (2019). New Zealand hospital stroke service provision: A national survey. Journal of Neurology, Neurosurgery \& Psychiatry, 90(e7) A33-A34. https://doi. org/10.1136/jnnp-2019-anzan.91

Willan, J., King, A. J., Jeffery, K., \& Bienz, N. (2020). Challenges for NHS hospitals during covid-19 epidemic. BMJ, 368, m1117. https://doi. org/10.1136/bmj.m1117

Yeo, J., Zhou, L., \& Ratnasabapathy, Y. (2016). Stroke care delivery at North Shore Hospital, Waitemata District Health Board 2014. New Zealand Medical Journal, 129(1431), 67-79. 